\title{
TV/Series
}

19 | 2021

Perfectionnisme et séries télévisées. Hommage à Stanley Cavell (1926-2018)

\section{Exercices spirituels et séries télévisées. The Walking Dead, Game of Thrones, Dexter}

\section{Hugo Clémot}

\author{
(2) OpenEdition \\ Journals \\ Édition électronique \\ URL : https://journals.openedition.org/tvseries/5305 \\ DOI : $10.4000 /$ tvseries.5305 \\ ISSN : 2266-0909 \\ Éditeur \\ GRIC - Groupe de recherche Identités et Cultures
}

Référence électronique

Hugo Clémot, "Exercices spirituels et séries télévisées. The Walking Dead, Game of Thrones, Dexter », TV/Series [En ligne], 19 | 2021, mis en ligne le 06 mai 2021, consulté le 14 mai 2021. URL : http:// journals.openedition.org/tvseries/5305; DOI : https://doi.org/10.4000/tvseries.5305

Ce document a été généré automatiquement le 14 mai 2021.

\section{c)}

TV/Series est mis à disposition selon les termes de la licence Creative Commons Attribution - Pas d'Utilisation Commerciale - Pas de Modification 4.0 International. 


\title{
Exercices spirituels et séries télévisées. The Walking Dead, Game of Thrones, Dexter
}

\author{
Hugo Clémot
}

\section{Introduction : Étudier les séries télévisées après Stanley Cavell}

1 En tant que précurseur de la philosophie contemporaine du cinéma, Stanley Cavell a dû surmonter un certain nombre d'obstacles épistémologiques qui empêchaient, à l'époque où il écrivait $L a$ projection $d u$ monde ${ }^{1}$ (1971 pour la première édition), que les films hollywoodiens des années 1930-40 soient tenus pour des œuvres d'art dignes d'un intérêt philosophique. Ces difficultés sont abordées dans le premier chapitre, où Cavell les identifie et propose des moyens de les dépasser. Or, il est remarquable qu'y soient évoquées au moins deux des caractéristiques des séries télévisées qui ont pu retarder leur étude sérieuse, sinon empêcher qu'on réfléchisse sur elles, à savoir la question de l'auteur et celle du mode - commercial - de leur production. Ce ne sont cependant là que certaines des caractéristiques abordées: de même qu'il se dit troublé par les nouvelles manières de regarder des films dans les salles, au début des années 1970, Cavell réfléchit aussi à la question de savoir comment se rapporter correctement à des films qu'il n'a, pour certains, vus qu'une seule fois, trente à quarante ans auparavant. Dans la mesure où il se trouve ainsi empêché de décrire ces films de façon exhaustive, la difficulté de sa situation est assez analogue à celle des études sérieuses sur les séries télévisées.

2 Il se pourrait que les réponses proposées par Cavell à ces questions au moment où s'invente avec lui la philosophie contemporaine du cinéma vaillent aussi en tant que réponses aux difficultés qui ont pu empêcher aussi longtemps et empêchent encore de réfléchir sérieusement sur les séries télévisées. En effet, Cavell énonce des idées qui ne semblent $a$ priori pas sans rapport avec les problèmes posés par le succès contemporain 
des séries télévisées quand il affirme, par exemple, à propos du débat autour de l'auteurisme que «ce n'est ni un romantisme de l'anonymat, ni un romantisme de l'individualité qui vont rendre compte du pouvoir que le cinéma a, ou a eu, pour nous $^{2}$ », ou lorsqu'il rejoint Erwin Panofsky en comparant la réalisation d'un film à la construction d'une cathédrale et remarque que

si l'on définit l'art commercial comme tout art non produit, de prime abord, dans le but de satisfaire le besoin créateur de l'artiste, mais visant à satisfaire les exigences d'un mécène ou d'un public, il faut préciser que l'art non commercial est une exception plutôt que la règle, exception récente, de plus, et pas toujours heureuse ${ }^{3}$.

Cette proximité pourrait même conduire à se demander, malgré les différences évidentes entre séries et films traditionnels, si les séries télévisées ne seraient pas du cinéma continué par d'autres moyens.

Le but de cet article n'est cependant pas d'exposer les solutions aux problèmes soulevés par les séries télévisées que la lecture de Cavell pourrait nous suggérer, mais d'attirer l'attention sur un aspect du rapport entre le perfectionnisme cavellien et les séries télévisées. Plus précisément, je souhaite soutenir que regarder des séries peut transformer et améliorer nos façons de penser et d'agir d'une manière analogue à la transformation que devrait offrir la réflexion philosophique selon Stanley Cavell. Abondant dans le sens de Daniele Lorenzini quand il soutient que, pour Cavell, «le cinéma peut être considéré comme un véritable manuel d'exercices spirituels ${ }^{4}$ », j'entends donner quelques exemples d'exercices que l'on peut pratiquer à partir de certaines séries télévisées.

\section{Perfectionnisme, exercices spirituels et séries télévisées}

On crédite souvent Stanley Cavell d'avoir redécouvert, au cours de sa lecture des " comédies du remariage », le sens et l'importance du perfectionnisme moral de Ralph Waldo Emerson'. L'idée de perfectionnisme a une longue histoire que l'on peut rattacher à la tradition antique des exercices spirituels telle que Pierre Hadot, lecteur de Wittgenstein ${ }^{6}$ et de Cavell ${ }^{7}$, l'a étudiée ${ }^{8}$. Dans ce qui va suivre, j'aimerais montrer que nous pouvons pratiquer de tels exercices de transformation mentale et morale en nous aidant des séries télévisées.

\subsection{La philosophie comme transformation mentale : le perfectionnisme antique}

6 Cette conception de la philosophie comme activité de transformation mentale par laquelle on prend soin de son âme ne date en effet ni de Cavell, ni même d'Emerson : ce serait en fait la première à s'être imposée dans l'Antiquité'. Les philosophes antiques concevaient eux aussi cette transformation comme un moyen de s'améliorer soi-même. Ils considéraient que nous sommes tous réduits à une situation d'aliénation où nous ne sommes pas maîtres de nos pensées et de nos vies parce que ce sont les habitudes, les préjugés et le conformisme qui nous dominent. Pour se libérer, ils ne voyaient d'autre solution que de faire l'effort de penser par soi-même. Ils avaient conçu à cet effet des exercices à faire chaque jour, exercices dont la pratique régulière devait avoir pour 
effet d'augmenter la force et de donner une nouvelle forme à l'esprit du pratiquant : on a pu parler d'« exercices moraux ${ }^{10}$ » ou d'« exercices spirituels » à leur propos :

Par ce terme d'exercices spirituels, on entend toute manière de rencontrer Dieu, de méditer, de contempler, d'examiner sa conscience, de recevoir les sacrements, etc. De même en effet, que la promenade, la marche ou la course, sont des exercices physiques profitables à la santé du corps, de même on appelle exercices spirituels toute manière de préparer et de disposer l'âme pour, avec la grâce de Dieu : écarter de soi tous les attachements désordonnés, rechercher la volonté de Dieu pour moi, s'engager sur cette voie de salut ${ }^{11}$.

Michel Foucault les a appelées les « techniques de soi » :

les techniques de soi, qui permettent aux individus d'effectuer, seuls ou avec l'aide d'autres, un certain nombre d'opérations sur leur corps et leur âme, leurs pensées, leurs conduites, leur mode d'être; de se transformer afin d'atteindre un état de bonheur, de pureté, de sagesse, de perfection ou d'immortalité ${ }^{12}$.

\subsection{Exercices spirituels et expérience des séries télévisées}

On peut soutenir que l'expérience d'un visionnage quotidien des séries peut fonctionner comme un exercice spirituel dont la quotidienneté assurerait la transformation de potentialités mentales et morales latentes en capacités réelles. De même que l'exercice physique quotidien permet de donner une force et une forme nouvelle à nos corps, de même l'expérience ordinaire des séries télévisées serait-elle ainsi capable de renforcer et de transformer nos esprits. Suivant la recommandation cavellienne de reconnaître la dimension nécessairement autobiographique de l'expérience philosophique, je montrerai que c'est le cas à partir d'exemples tirés principalement de trois séries populaires comme The Walking Dead (Frank Darabont et Robert Kirkman, AMC, 2010-), Dexter (James Manos Jr., Showtime, 2006-2013), Game of Thrones (David Benioff, D. B. Weiss et R. R. Martin, HBO, 2011-2019).

\section{Revenir à soi, revenir au monde et s'ouvrir aux autres grâce aux séries télévisées}

9 Les exercices spirituels ont trois grands types d'effets : ils permettent de revenir à soi, de revenir au monde et de s'ouvrir aux autres. Pour chacun de ces trois effets, on peut décrire plusieurs exercices. On peut pratiquer ces exercices à l'aide de certaines séries télévisées et atteindre ces mêmes effets.

\subsection{Revenir à soi}

\subsubsection{L'ascèse}

Le premier exercice est ascétique : il s'agit de renforcer ou d'instituer la maîtrise de soi par le refus de certains plaisirs et l'épreuve de la douleur. Nombreux sont les films et les séries télévisées post-apocalyptiques qui prônent aujourd'hui une forme d'ascétisme. Dans ces œuvres, en effet, les personnages font l'expérience, et les spectateurs avec eux, de ce que le bonheur tient moins à leurs conditions matérielles d'existence qu'à leur état d'esprit : ils s'aperçoivent en effet souvent que leurs vies sont plus heureuses après l'apocalypse qu'elles ne l'étaient avant parce qu'ils savent désormais se réjouir de ce qu'ils ont, plutôt que de se plaindre de ce qu'ils n'ont pas ou 
plus et parce qu'ils ont appris à identifier leurs besoins réels et à renoncer à leurs désirs artificiels et vains.

Figure 1 : Dialogue entre Maggie Rhee et Rick Grimes dans le comics The Walking Dead (R. Kirkman, Compendium 3, 2014)

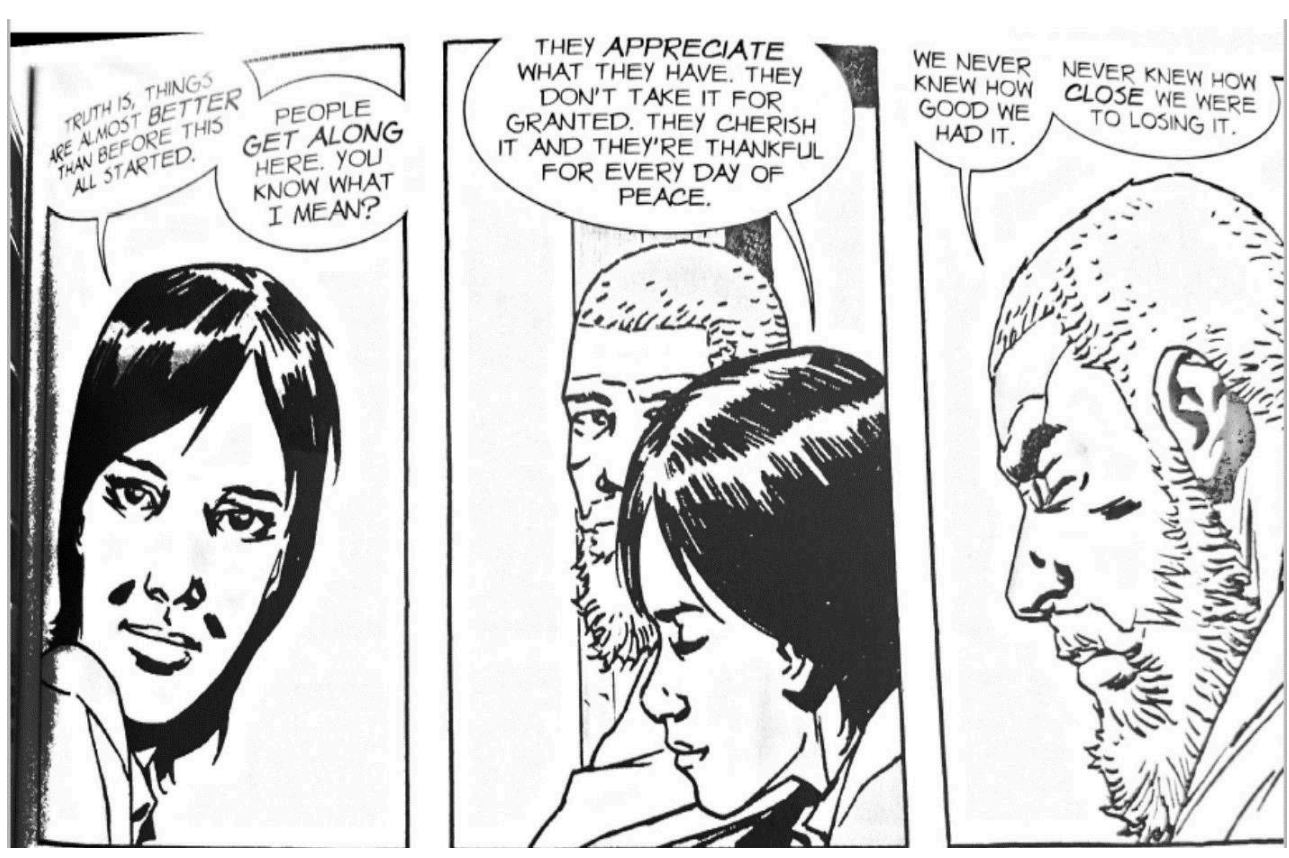

11 Comme le dit Maggie dans une vignette du comics The Walking Dead (voir figure 1) :

À vrai dire, les choses se passent presque mieux qu'avant que tout cela ne commence. Les gens [...] savourent ce qu'ils ont. Ils ne le tiennent pas pour acquis. Ils en apprécient la valeur et sont reconnaissants pour chaque jour de paix ${ }^{13}$.

En lui répondant «nous ignorions à quel point nous étions chanceux de vivre ainsi ", Rick s'aperçoit donc que les conditions du bonheur étaient déjà réunies avant l'apocalypse, que le monde ordinaire était un endroit où l'on pouvait vivre heureux, mais que le bonheur était manqué à cause d'une mauvaise façon de penser, d'une ignorance qui s'ignorait en tant que telle. Autrement dit, cette planche et, d'une façon générale, la série semblent nous indiquer que ce qui nous manque essentiellement pour être heureux, ce ne sont pas de meilleures conditions matérielles d'existence, mais une réforme de nos façons de voir et de concevoir le monde et nos rapports avec les autres, c'est-à-dire de philosophie.

\subsubsection{Le détachement}

Le deuxième exercice est celui du détachement : il faut que l'individu s'entraîne à se détacher de ce qui n'est pas lui, « en découvrant qu'il n'est pas ce qu'il croyait être, qu'il ne se confond pas avec les objets auxquels il s'était attaché14 ${ }^{14}$. Les séries télévisées présentent fréquemment des personnages traumatisés auxquels une seconde chance va être donnée, à condition qu'ils parviennent à se détacher de ce qu'on leur a fait, de ce qui est arrivé à leurs proches ou de ce qu'ils ont fait ou dit dans le passé ${ }^{15}$. Dans la série The Walking Dead, c'est notamment le cas du personnage de Daryl Dixon, l'un des plus populaires alors qu'il n'existe pas dans le comics. Daryl vient d'un milieu modeste marqué par l'alcoolisme et la violence. Enfant battu et abandonné à lui-même, son traumatisme lui vient aussi d'avoir erré enfant pendant neuf jours dans une forêt, sans 
que personne ne le recherche. Il ne le surmontera cependant qu'au douzième épisode de la saison 4 , en brûlant une cabane de forêt semblable à celle dans laquelle sa famille passait ses week-ends à s'enivrer, un rituel conçu pour se détacher de ce passé traumatisant, afin d'envisager l'avenir avec confiance. Dans une série télévisée postapocalyptique comme The Walking Dead, les personnages trouvent donc souvent leur salut dans le pouvoir de vivre seulement dans le présent, sans souffrir du passé qui ne les concerne plus, ni s'inquiéter de l'avenir qui est incertain et ne les concerne pas encore. On en trouve un autre exemple, dans un épisode particulièrement sombre ("Hearts Still Beating", S07E08) où l'espoir vient néanmoins à la fin quand un personnage incite un autre à savoir se réjouir du simple fait d'être en vie : " either your heart's beating, or it isn't ${ }^{16} »$. "[A]voir conscience qu'on vit est au nombre des plaisirs agréables par soi ${ }^{17}$ ", écrivait Aristote ; cet épisode nous rappelle l'existence du simple plaisir de se sentir exister.

\subsubsection{La méditation sur la mort ${ }^{18}$ et l'examen de conscience}

14 Cependant, regarder cette série où zombies et ennemis menacent constamment la vie des héros permet également de pratiquer un autre exercice, l'« exercice de la mort ${ }^{19}$ " qui consiste à avoir toujours à l'esprit la pensée de la possibilité de la mort afin d'intensifier quotidiennement l'expérience en laquelle consiste le fait d'avoir la chance de vivre encore pour quelque temps ${ }^{20}:$ « Persuade-toi que chaque nouveau jour qui se lève sera pour toi le dernier. Alors c'est avec gratitude que tu recevras chaque heure inespérée ${ }^{21}$.»

15 La joie avec laquelle on peut recevoir chaque moment que l'on a la chance de pouvoir vivre dépend de l'attention que l'on porte à l'idée que l'on va mourir et donc de l'attention à soi, de la conscience de soi. Pour atteindre cette conscience de soi qui conditionne la transformation mentale et morale à laquelle on doit aspirer, les écoles philosophiques de l'Antiquité recommandaient l'exercice quotidien de l'examen de conscience. Il s'agissait de se remémorer les fautes et les bonnes actions du jour en vue de se corriger. Un célèbre passage des Vers d'or de Pythagore ${ }^{22}$ en décrit les modalités :

Ne laisse le sommeil tomber sur tes yeux las

Avant d'avoir pesé tous les actes du jour :

En quoi ai-je failli? Qu'ai-je fait, quel devoir ai-je omis?

Commence par là et poursuis l'examen ; après quoi

Blâme ce qui est mal fait, du bien réjouis-toi.

16 Cette pratique de l'examen de conscience se retrouve systématiquement dans les épisodes de la série Dexter, la voix off du personnage principal exprimant ses doutes, ses regrets, mais aussi ses joies. Quand nous suivions la série, nous étions invités à faire chaque soir notre propre examen de conscience, à nous blâmer pour nos erreurs ou nos fautes et à nous réjouir pour nos bonnes actions.

Il est remarquable que l'institution de l'examen de conscience ait souvent été conçue comme étant reliée à celle de la direction spirituelle. Pierre Hadot nous apprend ainsi que Galien «conseillait en effet de se faire avertir de ses fautes par un homme âgé et expérimenté, et ensuite de s'examiner soi-même, matin et soir ${ }^{23}$.» Comment ne pas songer à ces discussions durant lesquelles Dexter, se parlant à lui-même, s'imagine être conseillé, blâmé ou félicitéé par Harry, son père adoptif? Quand on lit l'analogie proposée par Sénèque pour décrire l'examen de conscience: "Fais fonction d'accusateur de toi-même, puis de juge, en dernier lieu d'avocat ${ }^{25}$ ", comment ne pas 
songer aussi à la vigilance constante vis-à-vis de soi-même à laquelle Dexter a été entraîné depuis l'enfance et à la façon dont il a dû intérioriser le "Code d'Harry »? Mais si Harry assure la direction spirituelle de Dexter, alors peut-être le rôle joué par le personnage de Dexter est-il aussi d'assurer la direction spirituelle du spectateur.

\subsection{Revenir au monde}

\subsubsection{Le regard d'en haut}

L'un des exercices pour revenir au monde consiste à se transporter par la pensée en un point de vue tellement élevé qu'on puisse embrasser la totalité de la réalité, de façon synchronique comme diachronique. L'un des effets visés est d'éprouver la petitesse et l'insignifiance des affaires humaines. Depuis les astres, la terre n'est qu'un point où le luxe des riches est aussi ridicule que les guerres pour les frontières ${ }^{26}$.

Figure 2 : L'œil du générique de GoT (S01E01)

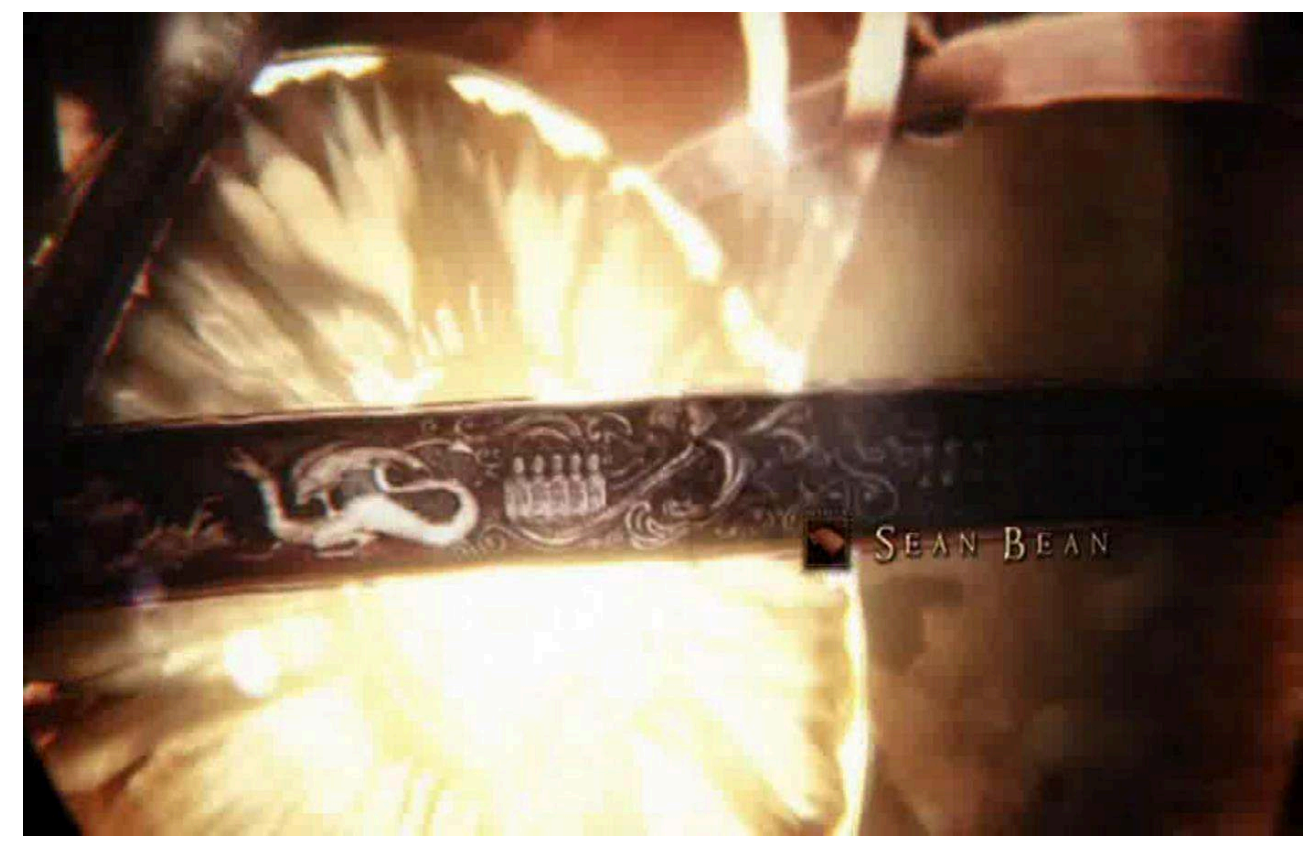


Figure 3 : Le regard d'en haut dans le générique de GoT (S01E01)

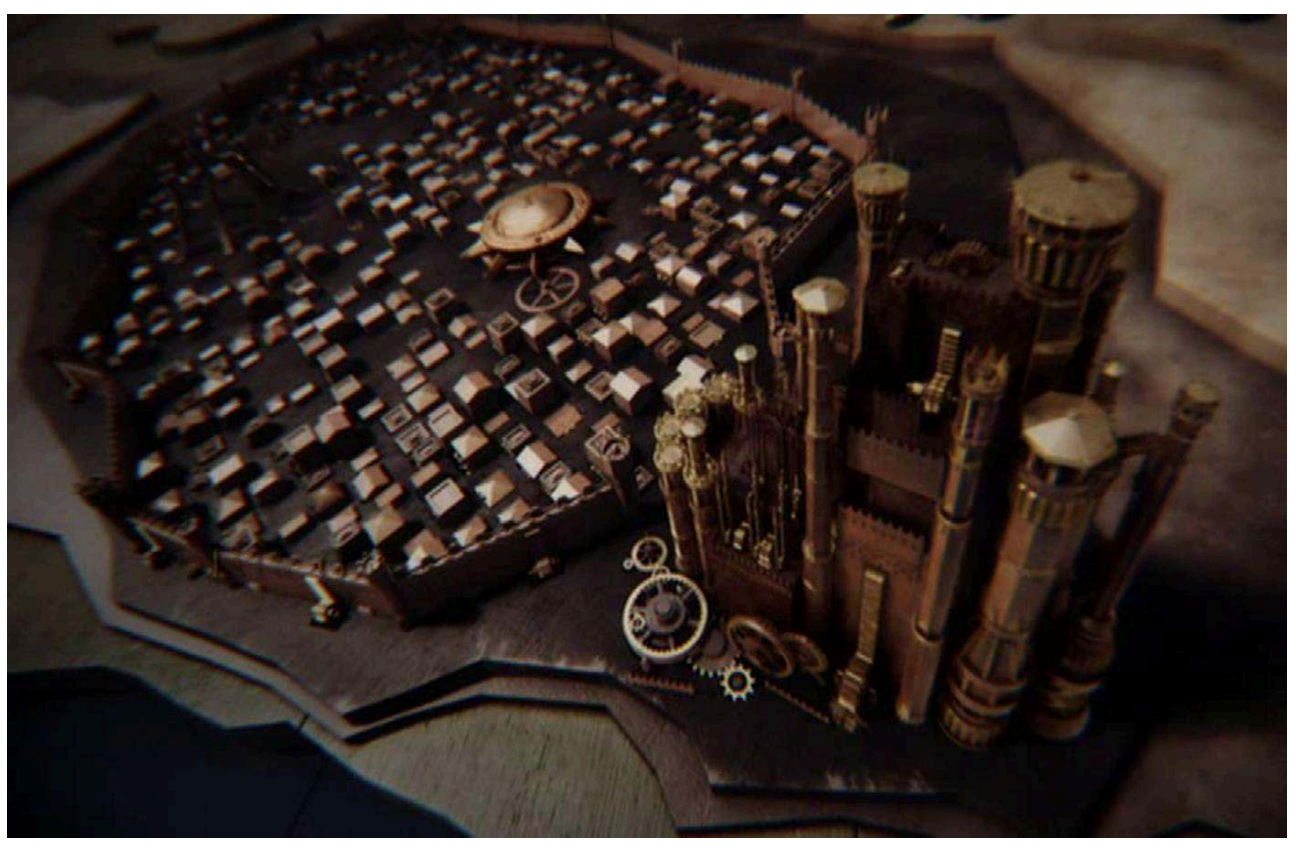

Il se trouve que la série la plus populaire des années 2010 aura systématiquement proposé dans son générique (voir figures 2 et 3 ) le point de vue d'un œil qui regarde d'en haut et se déplace sur la carte de sept royaumes ${ }^{27}$. Les villes qu'on y voit, quand l'œil s'arrête sur elles, semblent surgir du néant, sous l'effet d'une mécanique faite de gigantesques rouages et engrenages tout autant capable de les anéantir. S'il n'est pas absurde de penser que cette mécanique symbolise les passions et intrigues humaines qui fondent et animent les civilisations, alors il n'est peut-être pas dénué de sens de penser que ce générique nous rappelle que «les affaires humaines ne sont que mesquineries et néant ${ }^{28}$ ». On aura souvent vu également dans cette série des points de vue aériens depuis lesquels les esclaves de Yunkaï qui portent Daenerys en triomphe lorsqu'elle les a libérés en S03E10 (voir figure 4) où les "armées qui envahissent les territoires (voir figure 5) ne sont que des fourmis qui s'évertuent sur un étroit espace $^{29}$.» 
Figure 4 : Les esclaves vus d'en haut (GoT, S03E10)

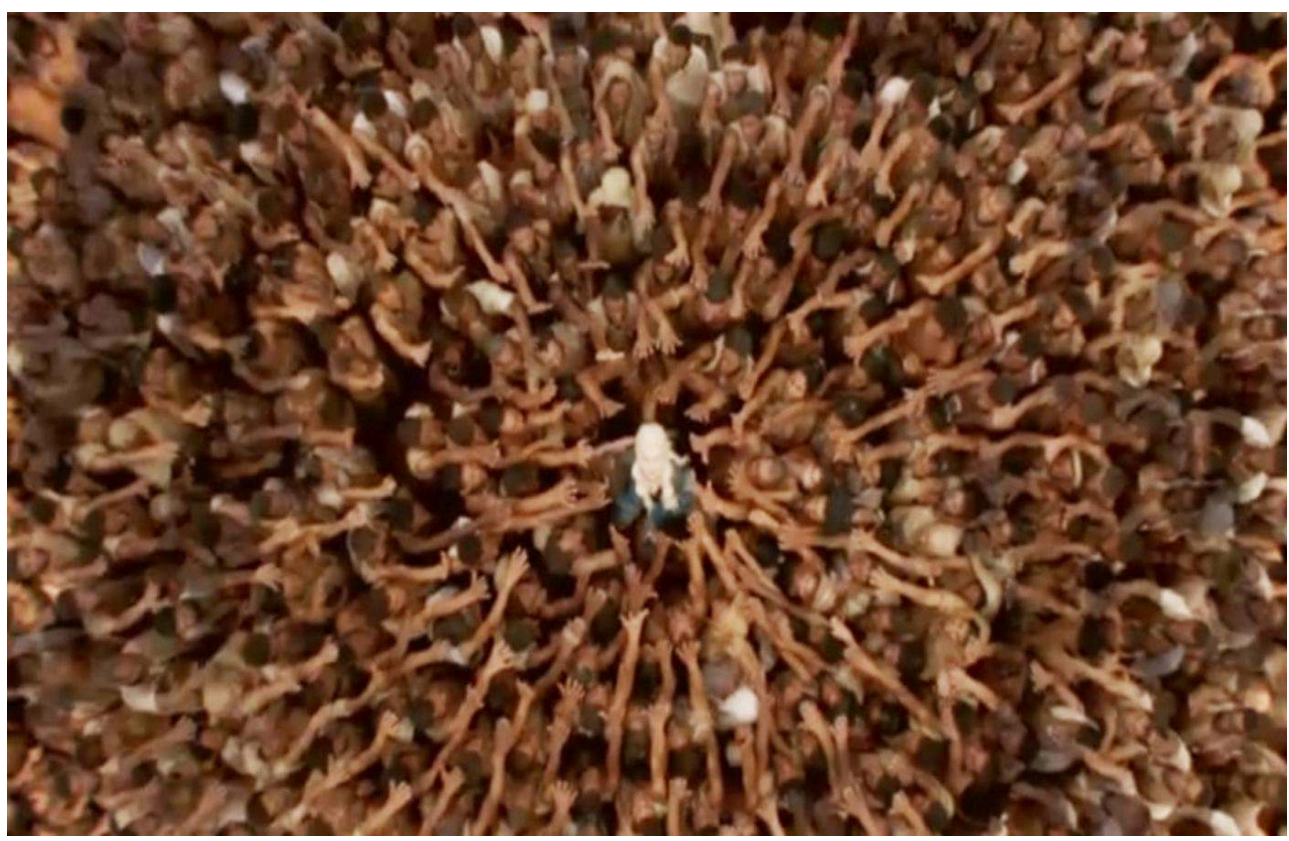

Figure 5 : Les armées vues d'en haut (GoT, S06E09)

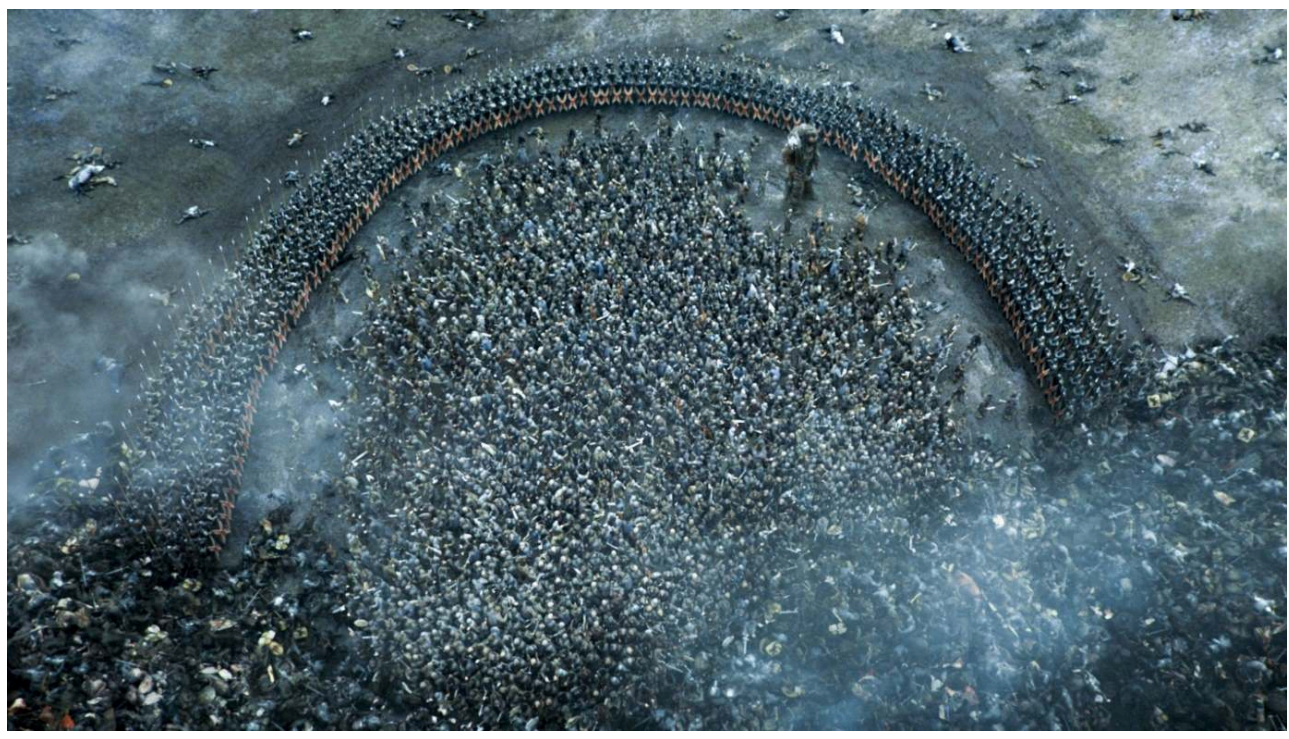

\subsubsection{La « vision de l'universelle métamorphose ${ }^{30}$ »}

Mais le rapport entre cet exercice spirituel et la série GoT me semble plus étroit encore si l'on se rappelle que «Regarder d'en haut, c'est regarder les choses dans la perspective de la mort ${ }^{31}$ ». En effet, s'il est vrai que le cinéma et les séries télévisées nous offrent le moyen d'identifier, d'analyser et de renverser ce qu'Emerson appelle les " seigneurs de la vie ${ }^{32}$ ", c'est-à-dire les catégories très générales qui structurent notre expérience à notre insu, les modes de vision ou encore les images dont nous sommes captifs, alors le succès des films apocalyptiques et post-apocalyptiques, parmi lesquels on peut compter une série d'horreur épidémique ${ }^{33}$ comme The Walking Dead, s'explique en partie parce qu'ils nous offrent un voyage sur les terres de la caducité ou de ce que 
Freud appelle, en un emprunt à Goethe, la "passagèretée ${ }^{34}$ ». La caducité est l'idée que tout ce qui existe est appelé à disparaître et tout ce qui vit à mourir un jour. Que la caducité règne sur nos esprits n'aurait certes rien d'étonnant en nos temps d'obsolescence programmée et de renouvellement constant du système des objets, d'insécurité économique, sociale, politique, mais aussi et surtout de disparition d'espèces végétales, animales, des lieux de nature, etc., une crise environnementale qui porte le nom de "réchauffement climatique ", mais que la série télévisée la plus populaire de la décennie appelait "l'hiver approche» ("Winter is coming.»). Trois grands faits narratifs frappants de Game of Thrones trouvent un écho étrangement inquiétant dans nos esprits d'individus vivant en ce début de $\mathrm{xxl}^{\mathrm{e}}$ siècle : la menace climatique, la mortalité des régimes politiques et la surmortalité des personnages principaux. La menace de l'hiver est évidemment la menace d'un dérèglement climatique porteur de conséquences catastrophiques, y compris quant à la question des réfugiés climatiques qui ne sont pas seulement représentés par le peuple des Sauvageons, mais aussi par les zombies ${ }^{35}$. La mortalité des civilisations $s^{36}$ et des régimes politiques nous rappelle, quant à elle, que nos institutions sociales et politiques démocratiques ne sont pas autre chose que nos manières de vivre et de penser ensemble et qu'elles en ont donc la force et la fragilité. Á en croire les réactions des spectateurs et spectatrices ${ }^{37}$, l'une des forces de la série The Handmaid's Tale (Bruce Miller, Hulu, 2017-) a ainsi été de permettre la prise de conscience de la fragilité des droits des femmes et de l'importance du combat féministe.

21 La mort des personnages principaux, enfin, ne nous rappelle pas seulement que l'attente de la mort est l'horizon indépassable de nos vies ("Valar morghulis»). La mort d'un « personnage $P_{0} V^{38}$ » est en effet aussi la disparition d'un point de vue, d'une world view ou encore d'une " conception du monde " et l'occasion d'un travail de deuil qui est analogue au travail philosophique, le danger étant pour nous de devenir, comme les compagnons de promenade de Freud dans son texte sur la " passagèretée ${ }^{39}$ ", insensibles aux beautés du monde en ne le voyant plus que sous l'aspect de sa nécessaire caducité.

L'une des idées que la série GoT nous fait le mieux intérioriser est donc bien celle de l'essentielle caducité ou passagèreté du monde. Or, en nous faisant voir le présent comme s'il était déjà passé, en nous donnant à contempler la façon dont les choses se transforment les unes dans les autres, la série nous fait pratiquer un autre exercice : la vision de l'universelle métamorphose qui conduit à la méditation sur la mort.

L'une des formes particulières que peut prendre cet exercice de la vision de l'universelle métamorphose est décrite par Marc-Aurèle dans les termes suivants: "Observe chaque objet et imagine-toi qu'il est en train de se dissoudre, qu'il est en pleine transformation, en train de pourrir et de se détruire ${ }^{40}$. " Appliquée à nos villes, cette démarche imaginaire nous pousse à nous représenter les quartiers les plus denses architecturalement et les plus animés comme désertés, détruits et laissés en friche (voir figure 6). Appliquée au corps et au comportement humains, cette démarche imaginaire nous conduit à l'image d'un homme sans ses qualités, mais avec tous ses défauts (voir figure 7), un être lent, peu conscient, amnésique, stupide, vorace, violent, laid, malodorant, grégaire, « en train de pourrir et de se détruire ${ }^{41}$ », etc. Il n'est pas difficile de retrouver l'iconographie de The Walking Dead. 
Figure 6 : L'entrée dans Atlanta (The Walking Dead, S01E01)

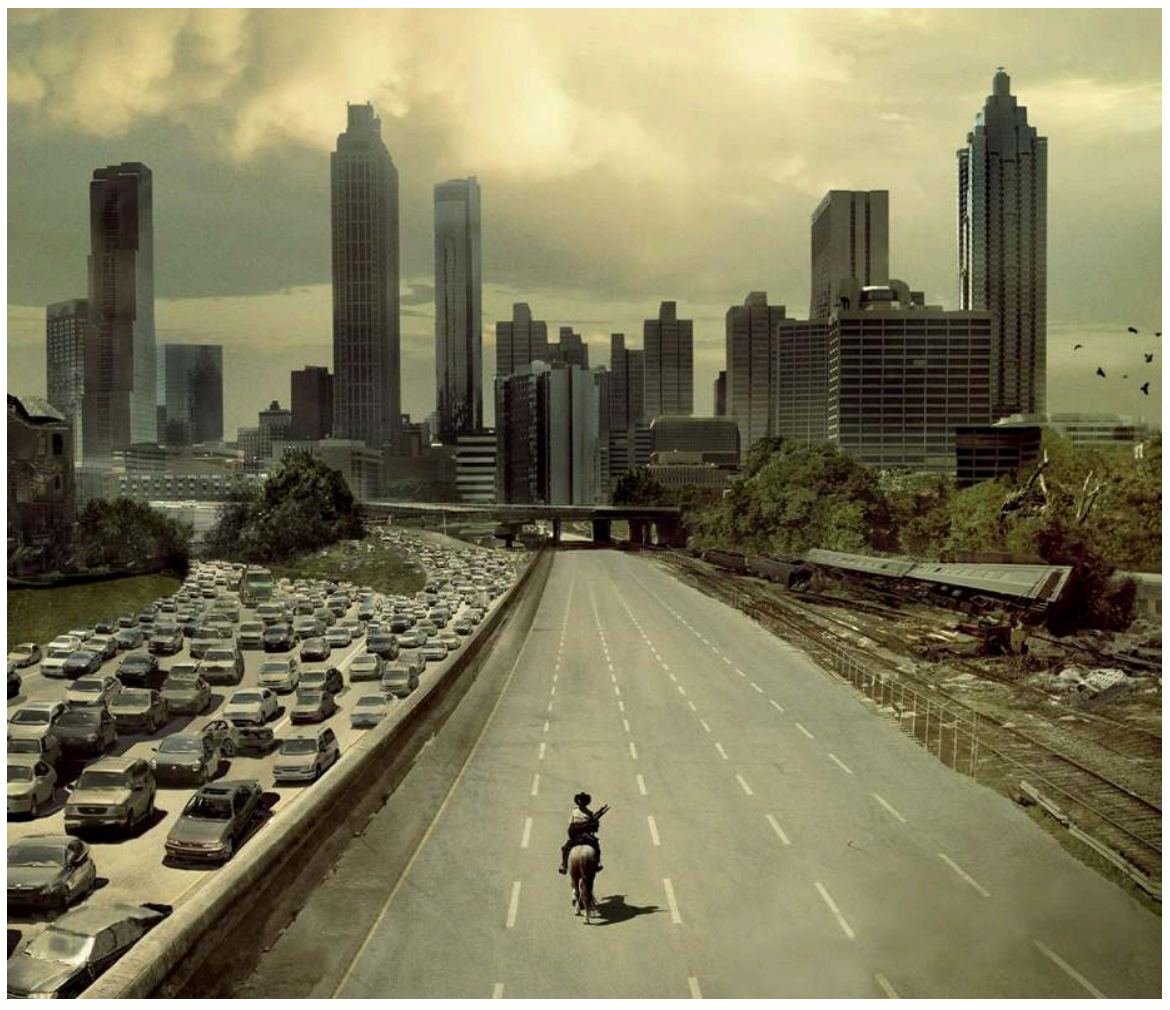

Figure 7 : La fille au vélo (The Walking Dead, S01E01)

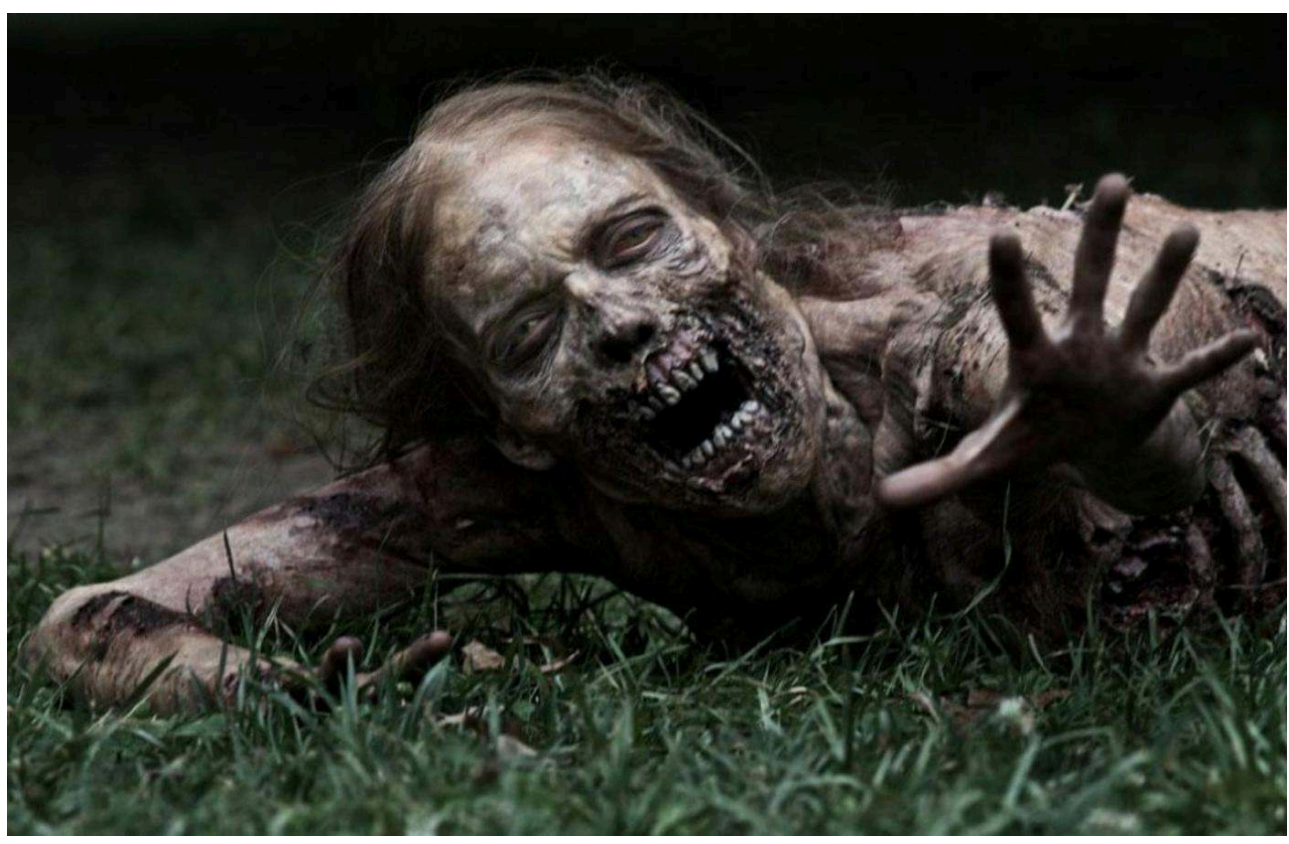

\subsubsection{La préméditation des maux}

24 Cet exercice de vision de l'universelle mobilité peut être pratiqué dans le cadre d'un autre exercice plus général : la préméditation des maux. Exercice de préparation aux épreuves, la prévision des difficultés à venir était en effet préconisée par les stoïciens, non seulement pour «amortir le choc de la réalité », mais aussi pour conserver le 
contrôle sur son intention morale et ainsi la tranquillité de l'âme, en se rappelant qu'un mal futur n'est pas un mal tant qu'il n'est pas présent et que les «les événements, comme la maladie, la pauvreté et la mort, que les autres hommes perçoivent comme des maux, ne sont pas des maux, puisqu'ils ne dépendent pas de nous ${ }^{42} »$. On peut donc supposer que regarder des séries post-apocalyptiques comme The Walking Dead et son spin-off Fear The Walking Dead (Robert Kirkman et Dave Erickson, AMC, 2015-) permet de s'entraîner à voir à l'avance les maux que les changements naturels et sociaux qui s'annoncent nous feront endurer. Conjugué à la menace permanente que font peser les zombies sur la vie des personnages, cet exercice peut permettre de renforcer l'effet de "la pensée de la mort imminente ${ }^{43}$ » en rehaussant la valeur de chaque action que nous avons la chance de pouvoir encore vivre avant la fin du monde tel que nous le connaissons.

\subsection{S'ouvrir aux autres}

Certains exercices spirituels dépendent d'un rapport à autrui qui passe d'abord, à suivre Pierre Hadot, par l'institution de la direction spirituelle ${ }^{44}$. Cette manière régulière d'assurer l'éducation morale d'un adulte s'inscrit dans une relation hiérarchique de maître à disciple et poursuit une double finalité: "permettre au disciple de prendre conscience de lui-même » et l'aider "à faire les choix particuliers raisonnables, dans la vie de tous les jours ${ }^{45}$."

\subsubsection{Prendre conscience de soi-même}

26 J'ai cru retrouver plus haut cette institution dans la relation entre Dexter et son père adoptif. Je souhaiterais explorer ici l'hypothèse laissée en suspens, à savoir que Dexter soit pour le spectateur un directeur spirituel. On sait que le personnage de Dexter est celui d'un sociopathe, c'est-à-dire quelqu'un qui ne parvient plus à éprouver d'émotions, depuis qu'il a assisté, étant enfant, à l'assassinat de sa mère, mais qui a appris à imiter les comportements émotionnels des autres pour survivre. Si Jean-Pierre Esquenazi a raison de penser que la vérité de la fiction lui vient de son pouvoir de paraphraser le réel, la paraphrase étant ce à quoi l'on a affaire « quand l'appropriation du récit par un destinataire [le] conduit à comprendre l'univers fictionnel comme [...] exemplifiant [...] une part du monde réel ${ }^{46} »$, alors on peut soutenir que Dexter paraphrase cette partie de nos existences qui relève du phénomène de la normopathie, cette

tendance pathologique à s'immerger dans la normalité et les vertus acceptables, une obéissance timorée qui élimine toute étincelle de pensée indépendante ou des émotions profondes comme la colère [...] ou l'empathie envers ceux qui souffrent ${ }^{47}$.

En effet, tout est fait dans la série pour que le spectateur s'aperçoive que le sentiment de vide dont ne cesse de parler Dexter lui est familier ${ }^{48}$. Accompagner Dexter au quotidien, c'est faire l'expérience de ce vide, de cette difficulté à atteindre l'autre que nous ressentons d'ordinaire et que nous avons appris à cacher sous des apparences de normalité. Or, à force d'étouffer nos sentiments, nous pouvons en venir à ne plus pouvoir éprouver d'empathie pour autrui : il suffit alors que l'autre nous gêne, nous dérange dans nos efforts pour cacher aux autres et nous cacher à nous-mêmes notre propre vide, notre propre vanité, pour que l'envie, la jalousie et la haine impuissante se développent et se transforment en désir d'infliger aux autres des souffrances brutales. 
De ce point de vue, la série Dexter permettrait aux destinataires qui y voient une paraphrase d'une partie de leur existence de se donner des représentations personnelles du drame qui les a conduits à cette normopathie ${ }^{49}$.

\subsubsection{Choisir au quotidien}

29 Mais la série ne permet pas seulement au spectateur de prendre conscience de luimême. En nous montrant Dexter composer avec son handicap émotionnel dans les situations sociales les plus ordinaires de la vie quotidienne (relations avec une mère divorcée, avec le père des enfants de sa compagne, relations de voisinage, relations professionnelles, repas d'anniversaire de son fils, etc.), la série nous aide aussi «à faire les choix particuliers raisonnables, dans la vie de tous les jours $»^{50}$. Par exemple, à la fin du quatrième épisode de la troisième saison, Dexter fait une demande en mariage couronnée de succès. Alors que la scène se conclut par une embrassade, la voix off de Dexter tire la morale de cette histoire (voir figure 8) : «Bien des acteurs restent dans l'ombre, sans jamais connaître les feux de la rampe. Mais si on aiguise son art, qu'on le travaille assidûment, on peut obtenir le rôle de toute une vie.» Cette leçon peut se comprendre ainsi : celui qui craint de ne pas savoir se comporter correctement parce qu'il craint de ne pas pouvoir éprouver les émotions attendues dans les situations sociales ne doit pas fuir pour autant la socialité pour se réfugier dans la solitude; ce n'est qu'en s'entraînant à jouer le jeu social, même si l'on ne fait que simuler au départ, que l'on parviendra à se comporter et à s'émouvoir spontanément tout en restant fidèle à soi-même. Il est clair que l'émotion suscitée par cette scène lacrymogène ne peut que faciliter la remémoration de cette leçon de sagesse.

Figure 8 : La demande en mariage (Dexter, S03E04)

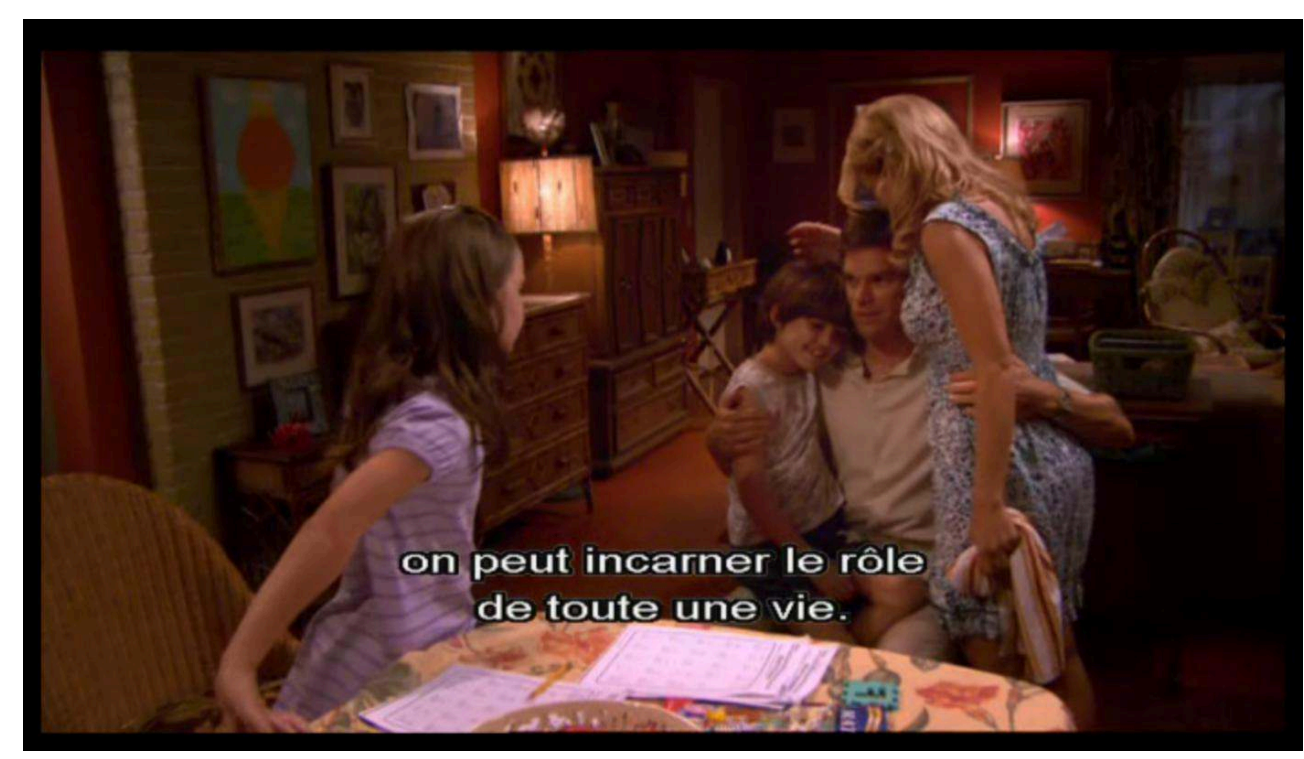

\subsubsection{La figure du sage}

Que Dexter soit une sorte de directeur spirituel du spectateur pourra être contesté : n'est-il pas qu'un personnage de fiction? Les pouvoirs dont il dispose n'en font-ils pas d'ailleurs une espèce de superhéros ${ }^{51}$, un être dont les perfections ne sauraient être atteintes? 

dernier exercice spirituel cité par Pierre Hadot et que le spectateur ordinaire de séries télévisées pratique spontanément : il s'agit de se poser la question "Que ferait Dexter (ou Buffy ${ }^{52}$ ou Jack Bauer) dans telle ou telle circonstance? » comme les philosophes antiques se demandaient "Que ferait le sage dans telle ou telle circonstance ${ }^{53}$ ? » En effet, P. Hadot note ainsi à propos des philosophes stoïciens :

Cette figure idéale du sage, le philosophe stoïcien sait qu'il ne pourra jamais la réaliser, mais elle exerce pourtant sur lui son attrait, provoque en lui l'enthousiasme et l'amour, lui fait entendre un appel à vivre mieux, à prendre conscience de la perfection qu'il s'efforce d'atteindre ${ }^{54}$.

Autrement dit, le sage n'a jamais été conçu comme un être immanent, mais plutôt comme un être transcendant, un idéal censé servir de norme à atteindre pour permettre à chacun d'aller le plus haut et le plus loin possible dans sa démarche de perfectionnement : comme l'a remarqué Arnold Davidson ${ }^{55}$, la philosophie est l'amour de cette sagesse transcendante, régulatrice. Mais il n'est pas difficile de reconnaître, derrière cette figure d'un sage transcendant, nos personnages de série télévisée préférés. Et si la philosophie est l'amour d'une telle sagesse, alors on pourra tenir notre attachement pour de tels personnages de fiction comme " philosophique ».

\section{Conclusion}

J'espère avoir montré que les séries télévisées peuvent donc bien être considérées comme des «manuels » d'exercices spirituels, non seulement parce qu'il est désormais aisé, grâce à internet et au cloud, d'avoir toujours «à portée de main » l'extrait, l'épisode, voire la série qui contient les exercices spirituels qui nous font du bien en nous rendant meilleurs, mais aussi par analogie avec le genre littéraire dont relève un texte comme le Manuel d'Epictète, les Pensées pour moi-même de Marc-Aurèle ou la Lettre à Ménécée d'Épicure.

34

À l'instar d'Épicure incitant Ménécée à soigner ses maux par la philosophie, il ne serait donc pas absurde de prescrire de regarder des séries télévisées à qui veut s'améliorer ${ }^{56}$. Mais, comme y insiste le natif de Samos auprès de son ami, se contenter de regarder une série ne suffit pas davantage à aller mieux que de se contenter de lire une lettre : ce serait en effet attribuer aux images et aux textes des pouvoirs médicinaux tels que l'on puisse songer à se soigner par la seule lecture d'une Fable de La Fontaine ${ }^{57}$. La sériethérapie réclamerait alors que l'on sache utiliser les séries de la bonne manière, que l'on définisse les éléments de sa posologie. Il est sûr que l'efficacité de ces exercices dépendrait du temps pris par le spectateur pour réfléchir, seul ou avec des amis, aux leçons qu'il pourrait tirer de ses expériences audiovisuelles. Autrement dit, pratiquer des exercices spirituels avec les séries télévisées supposerait certainement de s'engager dans un travail de « lecture » et d'écriture cinéthique ${ }^{58}$ sur les séries qui serait analogue à celui accompli par Cavell sur le cinéma tout au long de sa vie afin de répondre à l'appel d'Henry James : «Essayez d'être l'un de ces gens à qui rien n'échappe, pour qui rien ne se perd ${ }^{59}$. » Bref, s'aider des séries télévisées pour ne pas passer à côté de son expérience ordinaire et devenir meilleur suppose aussi de savoir détourner ses yeux de l'écran pour lire de la philosophie et en particulier les œuvres de Stanley Cavell. 


\section{BIBLIOGRAPHIE}

Allouche, Sylvie, LAUgier, Sandra, éds., Philoséries. Buffy. Tueuse de vampires, Paris, Bragelonne, 2014.

ARISTOTE, Éthique à Nicomaque, traduction Jules TRICOT, Paris, Vrin, coll. « Bibliothèque des textes philosophiques », 1990.

CAVELL, Stanley, La projection du monde. Réflexions sur l'ontologie du cinéma [1e éd. : 1971], présentation par Hugo CLÉMOT, trad. franç. par Chistian FOURNIER, Paris, Vrin, coll. « Philosophie au présent », 2019.

CAVELL, Stanley, À la recherche du bonheur. Hollywood et la comédie du remariage, [1 $\mathrm{e}^{\mathrm{e}}$ éd. : 1981], préface de Sandra LAUGIER, trad. franç. par Chistian FOURNIER et Sandra LAUGIER, Paris, Vrin, coll. «Philosophie du présent », 2017.

CAVELL, Stanley, Le cinéma nous rend-il meilleurs ?, éd. Élise DOMENACH, trad. franç. par Christian FOURNIER et Élise DOMENACH, Paris, Bayard, 2003.

CLÉMOT, Hugo, Cinéthique, Paris, Vrin, coll. « La vie morale », 2018.

CLÉMOT, Hugo, « La fin de Lost, le paradoxe des séries et l'expérience de la perte », TV/Series, Hors séries 1, 2016, http://journals.openedition.org/tvseries/1710.

CLÉMOT, Hugo, « Une lecture de Dexter », Implications philosophiques; volet (1), 4 mars 2013, https://www.implications-philosophiques.org/semaines-thematiques/philosophie-des-series/ une-lecture-de-dexter-1/, volet (2), 6 mars 2013, https://www.implications-philosophiques.org/ semaines-thematiques/philosophie-des-series/une-lecture-de-dexter-2/, et volet (3), 8 mars 2013, https://www.implications-philosophiques.org/semaines-thematiques/philosophie-desseries/une-lecture-de-dexter-3/.

CLÉMот, Hugo, « Une lecture des films d'horreur épidémique », Tracés, n² 21, 2011/2, p. 167-184, http://traces.revues.org/5209.

CLÉMOT, Hugo, "Émotions, philosophies analytique et post-analytique du cinéma ", Penser les émotions. Cinémas, séries, nouvelles images, éd. Martin BARNIER, Isabelle LE CORFF et Nedjma MOUSSAOUI, Paris, L’Harmattan, coll. « Champs visuels », 2016, p. 215-229.

DAVIDSON, Arnold, « Foucault, le perfectionnisme et la tradition des exercices spirituels ", in La voix et la vertu. Variétés du perfectionnisme moral, éd. Sandra LAUGIER, Paris, PUF, coll. « Éthique et philosophie morale », 2010, p. 449-467.

DESCOMBES, Vincent, La Denrée mentale, Paris, Minuit, 1995.

ESQUENAZI, Jean-Pierre, Éléments pour l'analyse des séries, Paris, L'Harmattan, coll. « Champs visuels », 2017.

ESQUENAZI, Jean-Pierre, La vérité de la fiction. Comment peut-on croire que les récits de fiction nous parlent sérieusement de la réalité ?, Paris, Hermès-Lavoisier, 2009.

FOJAS, Camilla, Zombies, Migrants and Queers. Race and Crisis Capitalism in Pop Culture, Chicago, University of Illinois Press, 2017.

FOUCAULT, Michel, L'herméneutique du sujet. Cours au Collège de France (1981-1982), Paris, GallimardSeuil, coll. « Hautes Études », 2001. 
FREUD, Sigmund, « Passagèreté » [1 $1^{\mathrm{e}}$ éd. : 1915], trad. franç. par Janine ALTOUNIAN, André BOURGUIGNON, Pierre COTET et Alain RAUZY, in CEuvres complètes. Psychanalyse, vol. XIII, 1914-1915, Paris, PUF, 2005, p. 321-324.

GILLY, Patrice, Le cinéma, une douce thérapie, Lyon, Chronique sociale, 2015.

GUATTARI, Félix, « Le divan du pauvre », Communications, n²3, 1975, p. 96-103, https:// www.persee.fr/doc/comm_0588-8018_1975_num_23_1_1352.

HADOT, Pierre, «La philosophie comme éducation des adultes ", in La voix et la vertu. Variétés du perfectionnisme moral, éd. Sandra LAUGIER, Paris, PUF, coll. « Éthique et philosophie morale », 2010, p. 439-447.

HADOT, Pierre, Wittgenstein et les limites du langage, Paris, Vrin, coll. « Bibliothèque d'Histoire de la Philosophie », 2004.

HADOT, Pierre, Exercices spirituels et philosophie antique, Paris, Albin Michel, 2002.

HADOT, Pierre, « La figure du sage dans l'Antiquité », in Études de philosophie ancienne, Paris, Les Belles Lettres, 1998, p. 232-257.

HADOT, Pierre, Qu'est-ce que la philosophie antique ?, Paris, Gallimard, coll. « Folio essais », 1995.

KAWAL, Jason, « Should We Do What Buffy Do?», in Buffy the Vampire Slayer and Philosophy, ed. James B. South, Chicago-LaSalle, Open Court, 2003, p. 149-159.

KIRKMAN, Robert, The Walking Dead, comics, Compendium 3, 2014.

LEITE, Carolina Alfradique, «J'ai vécu : Glitterbug et la méditation sur la mort », Implications philosophiques, $1^{\mathrm{e}}$ septembre 2014, http://www.implications-philosophiques.org/actualite/une/ jai-vecu-glitterbug-et-la-meditation-sur-la-mort/.

LEMAIRE DE BRESSY, Virginie, Cinémathérapie par les dessins animés. Grandir et s'épanouir en famille, Escalquens, Dangles, 2019.

LÉVI-STRAUSS, Claude, « L'efficacité symbolique » [1e éd. : 1949], Anthropologie structurale I, chap. X, Paris, Pocket, 1997, p. 213-234.

LORENZINI, Daniele, « Le spectateur perfectionniste. Du cinéma comme alèthurgie de la vie quotidienne », communication au colloque international « Philosophie des salles obscures », organisé par Sandra LAUGIER, 2 juillet 2011, Université Paris 1 Panthéon-Sorbonne.

MUIR, John Kenneth, « The Killing Joke », in Dexter and Philosophy, Mind over Spatter, ed. Richard GREENE, George A. REICH and Rachel ROBISON-GREENE, Chicago-Lasalle, Open Court, 2011, p. 3-13.

PANOKSKY, Erwin, «Style et matière du septième art », in Trois essais sur le style, trad. franç. Bernard TURLE, Paris, Le Promeneur, 1996, p. 109-145.

PIVEN, Jerry S., « Dexter's Mirror », in Dexter and Philosophy. Mind over Spatter, ed. Richard GREENE, George A. REICH and Rachel ROBISON-GREENE, Chicago-LaSalle, Open Court, 2011, p. 77-88.

ROLET, Stéphane, Le Trône de fer ou le Pouvoir dans le sang, Tours, Presses Universitaires François Rabelais, 2014.

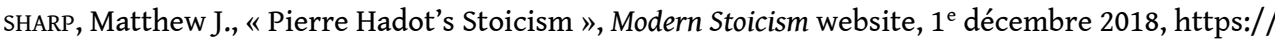
modernstoicism.com/pierre-hadots-stoicism-by-matthew-sharpe/. solomon, Gary, The Motion Picture Prescription, New York, Aslan Publishing, 1995. 
TISSERON, Serge, Comment Hitchcock m'a guéri. Que cherchons-nous dans les images ? [1 $1^{\mathrm{e}}$ éd. : 2003],

Paris, Fayard, coll. « Pluriel », 2011.

\section{NOTES}

1. Stanley Cavell, La projection du monde. Réflexions sur l'ontologie du cinéma [1e éd. : 1971], Paris, Vrin, coll. « Philosophie au présent », 2019.

2. Ibid., p. 37.

3. Erwin Panofsky, "Style et matière du septième art ", dans Trois essais sur le style, Paris, Le Promeneur, 1996, p. 138.

4. Daniele Lorenzini, «Le spectateur perfectionniste. Du cinéma comme alèthurgie de la vie quotidienne", communication au colloque international "Philosophie des salles obscures" organisé par Sandra Laugier, 2 juillet 2011, Université Paris 1 Panthéon-Sorbonne.

5. Stanley Cavell, À la recherche du bonheur. Hollywood et la comédie du remariage [1 ${ }^{\mathrm{e}}$ éd. : 1981], Paris, Vrin, coll. « Philosophie du présent », 2017.

6. Pierre Hadot, Wittgenstein et les limites du langage, Paris, Vrin, coll. «Bibliothèque d'Histoire de la Philosophie », 2004.

7. Pierre Hadot, «La philosophie comme éducation des adultes », in La voix et la vertu. Variétés du perfectionnisme moral, éd. Sandra Laugier, Paris, PUF, coll. « Éthique et philosophie morale », 2010, p. 439-447.

8. Voir Pierre Hadot, Exercices spirituels et philosophie antique, Paris, Albin Michel, 2002.

9. Voir Pierre Hadot, Qu'est-ce que la philosophie antique?, Paris, Gallimard, coll. "Folio essais », 1995.

10. Pour Paul Rabbow, les exercices moraux sont des «procédures ou actes déterminés, conçus pour s'influencer soi-même, poursuivis avec le but de produire un effet moral [...] toujours répétés ou |...] liés à d'autres actions pour former un ensemble méthodique. ", in Seelenführung: Methodik der Exerzitien in der Antike, München, Kösel, 1954, cité par Matthew J. Sharp, «Pierre Hadot's Stoicism », Modern Stoicism website, $1^{\mathrm{e}}$ décembre 2018, https://modernstoicism.com/ pierre-hadots-stoicism-by-matthew-sharpe/.

11. Ignace de Loyola, Exercices spirituels, Paris, Éditions religieuses, 2007, p. 25, cité par Arnold Davidson, «Foucault, le perfectionnisme et la tradition des exercices spirituels ", in La voix et la vertu. Variétés du perfectionnisme moral, éd. Laugier, op. cit., p. 452.

12. Michel Foucault, "Les techniques de soi », in Dits et écrits, Paris, Gallimard, 1994, vol. IV, p. 785, cité par Davidson, «Foucault, le perfectionnisme et la tradition des exercices spirituels », ibid., p. 452.

13. Voir R. Kirkman, The Walking Dead, comics, Compendium 3, 2014, p. 20.

14. Hadot, Qu'est-ce que la philosophie antique ?, op. cit., p. 292.

15. On peut penser à Six Feet Under (Alan Ball, HBO, 2001-2005), Lost (J. J. Abrams, Damon Lindelof et Jeffrey Lieber, ABC, 2004-2010), Dexter, The Leftovers (Damon Lindelof et Tom Perrotta, HBO, 2014-2017), mais aussi à Kidding (Dave Holstein, Showtime, 2018-) ou à The First (Beau Willimon, Hulu, 2018), mais la liste n'est pas exhaustive tant les scénaristes semblent fascinés par la question du deuil. Pour une réflexion sur ce thème à partir de Lost et de Dexter, voir Hugo Clémot, «La fin de Lost, le paradoxe des séries et l'expérience de la perte », TV/Series, Hors séries 1, 2016, http://journals.openedition.org/tvseries/1710 et "Une lecture de Dexter», Implications philosophiques, volet (1), 4 mars 2013, https://www.implications-philosophiques.org/semainesthematiques/philosophie-des-series/une-lecture-de-dexter-1/, volet (2), 6 mars 2013, https:// www.implications-philosophiques.org/semaines-thematiques/philosophie-des-series/une- 
lecture-de-dexter-2/, et volet (3), 8 mars 2013, https://www.implications-philosophiques.org/ semaines-thematiques/philosophie-des-series/une-lecture-de-dexter-3/.

16. «Soit ton cœur bat encore, soit il ne bat plus. "

17. Aristote, Éthique à Nicomaque, Paris, Vrin, coll. "Bibliothèque des textes philosophiques ", 1990, IX, 1170 b 1, p. 467.

18. Voir Michel Foucault. L'herméneutique du sujet, Paris, Gallimard, 2001, p. 458-459.

19. Voir Hadot, Qu'est-ce que la philosophie antique? op. cit., p. 296.

20. Pour un exemple analogue tiré du cinéma d'un auteur comme Derek Jarman, voir Carolina Alfradique Leite, «J'ai vécu : Glitterbug et la méditation sur la mort », Implications philosophiques, $1^{\mathrm{e}}$ septembre 2014, http://www.implications-philosophiques.org/actualite/une/jai-vecu-glitterbuget-la-meditation-sur-la-mort/

21. Horace, Odes, I, 11,7 ; Epitres, I, 4, 13, cité par Hadot, Qu'est-ce que la philosophie antique?, op. cit., p. 299.

22. Porphyre, Vie de Pythagore, § 40, et Epictète, Entretiens, III, 10, 3, cité par Hadot, ibid., p. 305.

23. Hadot, ibid., p. 306.

24. Par exemple, dans l'épisode 9 de la saison 5 , Dexter s'en prend au beau-père violent d'une jeune fille et lui fait quitter la ville pour toujours. Quand Dexter retourne à sa voiture, il s'entretient, de façon imaginaire, comme à son habitude, avec Harry qui le félicite pour ce qu'il vient de faire et lui dit qu'il s'est peut-être trompé sur son compte en pensant qu'il était un monstre incapable d'éprouver la moindre émotion pour autrui.

25. Sénèque, Lettres, 28, 10, cité par Hadot, Qu'est-ce que la philosophie antique ?, op. cit., p. 306.

26. Voir Hadot, ibid., p. 315, à propos de Sénèque, Questions naturelles, I, Prologue, 7-10.

27. Sur cet œil au centre d'une sorte de sphère armillaire, voir l'analyse du générique proposée par Stéphane Rolet dans Le Trône de fer ou le Pouvoir dans le sang, Tours, Presses Universitaires François Rabelais, 2014, p. 47-50.

28. Platon, Théétète, 173 e, cité par Hadot, Qu'est-ce que la philosophie antique ?, op. cit., p. 310.

29. Hadot, ibid., p. 315, à propos de Sénèque, Questions naturelles, I, Prologue, 7-10.

30. Hadot, ibid., p. 212.

31. Ibid., p. 316.

32. Pour une justification en relation avec la philosophie du cinéma de Stanley Cavell, voir Hugo Clémot, Cinéthique, Paris, Vrin, coll. « La vie morale », 2018, p. 78-88.

33. Voir Hugo Clémot, "Une lecture des films d'horreur épidémique », Tracés, n²1, 2011/2, p. 167-184, http://traces.revues.org/5209.

34. Sigmund Freud, "Passagèreté » [ 1 e éd. : 1915], Cuvres complètes. Psychanalyse, vol. XIII, 1914-1915, Paris, PUF, 2005, p. 321-324.

35. Voir Camilla Fojas, Zombies, Migrants and Queers. Race and Crisis Capitalism in Pop Culture, Chicago, University of Illinois Press, 2017.

36. Cf. l'exemple du Fléau de Valyria, évoqué notamment dans l'épisode 5 de la saison 5 par Jorah Mormont et Tyrion Lannister ; voir S. Rolet, Le Trône de fer, op. cit., p. 88.

37. Notamment lors des échanges qui ont suivi la conférence d'Aurélie Palud sur la série, le 11 avril 2018 à Tours, dans le cadre du cycle de conférences Serial Philo.

38. Voir la page consacrée à ce concept sur le wikisite La Garde de Nuit: https:// www.lagardedenuit.com/wiki/index.php?title=Personnages_PoV.

39. Freud, « Passagèreté », op. cit.

40. Marc-Aurèle, Pensées pour moi-même, $\mathrm{X}, 11$ et 18, cité par Hadot, Qu'est-ce que la philosophie antique ?, op. cit., p. 212.

41. Ibid.

42. Hadot, ibid., p. 213.

43. Ibid.

44. Ibid., p. 322-334. 
45. Ibid., p. 323.

46. Voir Jean-Pierre Esquenazi, La vérité de la fiction. Comment peut-on croire que les récits de fiction nous parlent sérieusement de la réalité ?, Paris, Hermès-Lavoisier, 2009, p. 155.

47. Voir Jerry S. Piven, « Dexter's Mirror », in Dexter and Philosophy. Mind over Spatter, éd. Richard Greene, George A. Reich et Rachel Robison-Greene, Chicago-LaSalle, Open Court, 2011, p. 80-81.

48. Voir Jean-Pierre Esquenazi, Éléments pour l'analyse des séries, Paris, L'Harmattan, coll. «Champs visuels ", 2017.

49. Pour une justification de cette idée qui emprunte une analyse de Serge Tisseron, dans Comment Hitchcock m'a guéri. Que cherchons-nous dans les images? [1 ${ }^{\mathrm{e}}$ éd. : 2003], Paris, Fayard, coll. «Pluriel», 2011, voir H. Clémot, «Émotions, philosophies analytique et post-analytique du cinéma ", in Penser les émotions. Cinémas, séries, nouvelles images, éd. Martin Barnier, Isabelle Le Corff et Nedjma Moussaoui, Paris, L'Harmattan, coll. « Champs visuels », 2016, p. 226-227.

50. Hadot, Qu'est-ce que la philosophie antique?, op. cit., p. 323.

51. Pour un exposé détaillé des ressemblances entre Dexter et les superhéros, voir John Kenneth Muir, « The Killing Joke », in Dexter and Philosophy, op. cit., p. 3-13,.

52. Voir Jason Kawal, « Should We Do What Buffy Do? ", in Buffy the Vampire Slayer and Philosophy, éd. James B. South, Chicago and LaSalle, Open Court, 2003, p. 149-159 et, en français, Philoséries. Buffy. Tueuse de vampires, éd. Sylvie Allouche et Sandra Laugier, Paris, Bragelonne, 2014.

53. Hadot, Qu'est-ce que la philosophie antique?, op. cit., p. 340.

54. Hadot, «La figure du sage dans l'Antiquité », in Etudes de philosophie ancienne, Paris, Les Belles Letres, 1998, p. 234.

55. Voir l'idée d'Arnold Davidson, «Foucault, le perfectionnisme et la tradition des exercices spirituels", in La voix et la vertu. Variétés du perfectionnisme moral, éd. Laugier, op. cit., p. 454-455.

56. Á en croire les partisans de la cinémathérapie, le cinéma aurait des vertus thérapeutiques et permettrait de révéler le meilleur de chacun de nous. Voir, en français, Virginie Lemaire de Bressy, Cinémathérapie par les dessins animés. Grandir et s'épanouir en famille, Escalquens, Dangles, 2019 ; Patrice Gilly, Le cinéma, une douce thérapie, Lyon, Chronique sociale, 2015 ; en anglais, Gary Solomon, The Motion Picture Prescription, New York, Aslan Publishing, 1995. Dans un tout autre contexte, Félix Guattari avait aussi décrit le cinéma comme «Le divan du pauvre »; voir son article « Le divan du pauvre », Communications, n²3, 1975, p. 96-103, https://www.persee.fr/doc/ comm_0588-8018_1975_num_23_1_1352.

57. Voir l'hilarante critique par Vincent Descombes d'une idée analogue de Claude Lévi-Strauss dans son fameux texte sur "L'efficacité symbolique » [1 éd. : 1949], in Anthropologie structurale I, chap. X, Paris, Pocket, 1997, p. 213-234 : Vincent Descombes, La Denrée mentale, Paris, Minuit, 1995, p. 143-150.

58. Voir Clémot, Cinéthique, op. cit.

59. Henry James, The Art of fiction [ $1^{\mathrm{e}}$ éd. : 1884], cité par Stanley Cavell dans Le cinéma nous rend-il meilleurs ?, éd. Élise Domenach, Paris, Bayard, 2003, p. 21.

\section{RÉSUMÉS}

La quotidienneté du visionnage des séries télévisées est l'un des traits spécifiques de l'expérience télésérielle les plus souvent relevés. Il est peut-être moins fréquent de soutenir que cette expérience peut fonctionner comme un exercice spirituel dont la pratique quotidienne assurerait 
la transformation de potentialités mentales et morales latentes en capacités réelles. De même que l'exercice physique permet de donner une force et une forme nouvelle à nos corps, de même l'expérience des séries télévisées serait-elle ainsi capable de renforcer et de transformer nos esprits. Mais si la philosophie est d'abord et surtout une activité de transformation de soi, alors on pourrait concevoir l'expérience télésérielle comme une expérience philosophique.

En remontant aux sources radicales du perfectionnisme moral de Ralph Waldo Emerson dans la tradition antique des exercices spirituels, l'article s'efforce de montrer que nous pouvons pratiquer ces exercices philosophiques de transformation mentale et morale avec les séries télévisées parce qu'elles peuvent notamment permettre de revenir à soi, de revenir au monde et de s'ouvrir aux autres. Si ces remarques devaient avoir un sens, elles ne seraient finalement qu'une autre façon de redire ce que Stanley Cavell nous a appris en nous incitant à nous servir des films comme d'un moyen de ne pas passer à côté notre expérience ordinaire.

One of the obvious specific traits of the television series experience is that we tend to watch it every day. It is perhaps less common to argue that this experience can function as a spiritual exercise whose daily practice would ensure the transformation of latent mental and moral potentialities into real capacities. Just as physical exercise gives our bodies a new strength and shape, so the experience of television series would be able to strengthen and transform our minds. But if philosophy is first and foremost an activity of self-transformation, then we could conceive of the television series experience as a philosophical experience.

By going back to the radical sources of Ralph Waldo Emerson's moral perfectionism in the ancient tradition of spiritual exercises, this article tries to show that we can practice these philosophical exercises of mental and moral transformation with television series because they can enable us to reconnect with ourselves, to the world and to others. If these remarks were to make sense, they would ultimately be just another way of repeating what Stanley Cavell taught us by encouraging us to use films as a way of not missing out on our ordinary daily experience.

\section{INDEX}

Mots-clés : Cavell Stanley, éthique, exercices spirituels, Hadot Pierre, perfectionnisme

Keywords : Cavell Stanley, ethics, Hadot Pierre, perfectionism, spiritual exercises

\section{AUTEUR}

\section{HUGO CLÉMOT}

Hugo Clémot est agrégé, docteur en philosophie et chargé de cours à l'Université de Tours. Spécialiste de philosophie du cinéma, associé au Centre de philosophie contemporaine de la Sorbonne (ISJPS). Il a publié les ouvrages suivants : Les jeux philosophiques de la trilogie Matrix (Vrin, 2011), La philosophie d'après le cinéma. Une lecture de La projection du monde de Stanley Cavell (Presses Universitaires de Rennes, 2014) et Cinéthique (Vrin, 2018). CEuvrant par ses articles et ses interventions à la diffusion des philosophies analytiques et post-analytiques du cinéma, il cherche aussi à contribuer à la création d'une philosophie des séries télévisées.

Hugo Clémot, laureate of the agregation, holds a $\mathrm{PhD}$ in philosophy and teaches at University of Tours. A specialist of the philosophy of cinema and a member of the Centre de philosophie contemporaine de la Sorbonne. He is the author of the following books: Les Jeux philosophiques de la trilogie Matrix (Vrin, 2011), La philosophie d'après le cinéma. Une lecture de La projection du monde de Stanley Cavell (Presses Universitaires de Rennes, 2014) et Cinéthique (Vrin, 2018). He aims at 
making philosophical (post) analyses of cinema more accessible and at creating a philosophy of TV series. 